\title{
REMARKS ON FIXED POINTS AND BOUNDARIES
}

\author{
W. A. $\mathbf{K I R K}^{1}$
}

\begin{abstract}
It is shown that three recent fixed point theorems of E. Chandler for nonexpansive mappings defined on compact subsets of strictly convex normed linear spaces hold in arbitrary normed linear spaces.
\end{abstract}

In [2], E. Chandler proves three fixed point theorems for nonexpansive mappings defined on compact subsets of strictly convex normed linear spaces. We show here that each of these holds without the strict convexity assumption.

We adopt the notation of [2]: $X$ denotes a compact subset of a normed linear space $E, \Delta^{\prime} X$ denotes the boundary of $X$ in $\overline{c o} X$, and $T: X \rightarrow X$ is nonexpansive $(\|T(x)-T(y)\| \leqslant\|x-y\|, x, y \in X)$.

THEOREM. In addition to the above, suppose one of the following conditions holds:

(1) $\|T(x)-T(y)\|<\|x-y\|$ for all $x, y \in \Delta^{\prime} X, x \neq y$.

(2) $T\left(\Delta^{\prime} X\right) \cap \Delta^{\prime} X=\varnothing$.

(3) There exists $y \in X$ such that $\|T(y)-x\|>\|y-x\|$ for all $x \in \Delta^{\prime} X$.

Then $T$ has a fixed point in $X$.

Proof. (1) For each $x \in X$ let $\omega(x)$ denote the set of points of $X$ which are limits of subsequences of $\left\{T^{n}(x)\right\}$. Then the sets $\omega(x)$ are compact and clearly $T$ : $\omega(x) \rightarrow \omega(x), x \in X$. Moreover if $D$ is any subset of $X$ which is minimal with respect to being nonempty, compact, and invariant under $T$, then

(i) $T$ is an isometry on $D[5]$;

(ii) $D=\omega(x)$ for each $x \in D[3]$.

Let

$$
\bar{y}=\left\{D \in 2^{X}: D \text { is minimal nonempty, compact, } T \text {-invariant }\right\}
$$

and let $\delta=\inf \{\operatorname{diam} D: D \in \bar{y}\}$. If $\delta=0$ then $\inf \{\|x-T(x)\|: x \in X\}=0$ and $T$ has a fixed point, so we suppose $\delta>0$. Select $D_{n} \in \overline{F_{F}} \operatorname{such}$ that $\operatorname{diam}\left(D_{n}\right) \rightarrow \delta$ as $n \rightarrow \infty$, and for each $n$ let $x_{n} \in D_{n}$. By compactness some subsequence $\left\{x_{n_{h}}\right\}$ of $\left\{x_{n}\right\}$ converges to a point $z \in X$. Let $\varepsilon>0$, fix $n$ so that $\left\|x_{n}-z\right\|<\varepsilon$, and select $w \in \omega(z)$. Then for some sequence $\left\{n_{1}\right\}_{i=1}^{\infty}$,

$$
T^{n_{1}}(z) \rightarrow w \text { as } i \rightarrow \infty,
$$

Received by the editors August 15, 1981 and, in revised form. April 12, 1982.

1980 Mathematics Subject Classification. Primary 47H10; Secondary 54H25.

Kel' words and phrases. Nonexpansive mappings, fixed points, normed linear spaces.

'Research supported by the National Science Foundation under grant MCS80-01604. 
and moreover some subsequence $\left\{T^{n_{i},}\left(x_{n}\right)\right\}_{j=1}^{\infty}$ of $\left\{T^{n_{i}}\left(x_{n}\right)\right\}_{i=1}^{\infty}$ converges to a point $u \in \omega\left(x_{n}\right)$ as $j \rightarrow \infty$. By nonexpansiveness,

$$
\|u-w\| \leqslant\left\|x_{n}-z\right\|<\varepsilon,
$$

proving that each point of $\omega(z)$ is within distance $\varepsilon$ of a point of $\omega\left(x_{n}\right)$. Therefore

$$
\delta \leqslant \operatorname{diam}(\omega(z)) \leqslant \delta_{n}+2 \varepsilon .
$$

Letting $n \rightarrow \infty$ and using the fact that $\varepsilon>0$ is arbitrary, we obtain

$$
\operatorname{diam}(\omega(z))=\delta .
$$

Next observe that $\overline{c o}(\omega(z)) \subset X$, for otherwise there would exist points $u, v \in \omega(z)$ with distinct points $u^{\prime}, v^{\prime} \in \operatorname{seg}[u, v] \cap \Delta^{\prime} X$. (1) then implies

$$
\begin{aligned}
\|T(u)-T(v)\| & \leqslant\left\|T(u)-T\left(u^{\prime}\right)\right\|+\left\|T\left(u^{\prime}\right)-T\left(v^{\prime}\right)\right\|+\left\|T\left(v^{\prime}\right)-T(v)\right\| \\
& <\left\|u-u^{\prime}\right\|+\left\|u^{\prime}-v^{\prime}\right\|+\left\|v^{\prime}-v\right\| \\
& =\|u-v\|
\end{aligned}
$$

which contradicts (i).

Since $\delta>0$ and $\overline{\operatorname{co}}(\omega(z))$ is compact, $\overline{\operatorname{co}}(\omega(z))$ must contain a point $u$ such that

$$
\sup \{\|u-x\|: x \in \overline{\operatorname{co}}(\omega(z))\}=r<\delta
$$

([4]). Let

$$
C=\{x \in X: \overline{\operatorname{co}}(\omega(z)) \subset B(x ; r)\}
$$

and note that $\left\{T^{n}(u)\right\} \subset C$ (because $T$ is nonexpansive and maps $\omega(z)$ onto $\omega(z)$ ). Thus if $s, t \in \omega(u)$, say $T^{n_{1}}(u) \rightarrow s$ and $T^{m_{1}}(u) \rightarrow t$ as $i \rightarrow \infty$, then for $m,>n_{i}$,

$$
\left\|T^{m},(u)-T^{n_{1}}(u)\right\| \leqslant\left\|T^{m_{1}-n_{i}}(u)-u\right\| \leqslant r .
$$

This implies diam $(\omega(u)) \leqslant r<\delta$, contradicting minimality of $\delta$.

Proof. (2) We suppose for convenience that $0 \in X, 0 \notin \Delta^{\prime} X$. Since $X$ is compact (2) implies $\operatorname{dist}\left(T\left(\Delta^{\prime} X\right), \Delta^{\prime} X\right)>0$, and thus for $t \in(0,1)$ sufficiently near $1, t T(x)$ $\in X$ for all $x \in \Delta^{\prime} X$. Also since $0 \in X$ and $T(x) \in X$ for all $x \in X, t T: X \rightarrow \overline{\operatorname{co}} X$. It is now possible to apply [1, Corollary 2] to the mapping $t T$ (taking $X=K$ and $\overline{c o} X=H)$ and obtain $x_{t} \in X$ such that $t T\left(x_{t}\right)=x_{t}$. Thus $\left\|x_{t}-T\left(x_{t}\right)\right\|=$ $(1-t)\left\|x_{t}\right\|$ yielding $\inf \{\|x-T(x)\|: x \in X\}=0$. Since $X$ is compact, this completes the proof.

For the proof of (3) we need the following very elementary fact: (Here $\partial_{K} B$ denotes the relative boundary of $B$ in $K$.)

Proposition 1 [6]. Let $X$ be a Banach space, let $K$ be a closed convex subset of $X$, and let $B$ be an open subset of $X$ with $B \cap K \neq \varnothing$. Suppose $\ddot{T}: \overline{B \cap K} \rightarrow K$ is a closed mapping which is a local contradiction on $D=B \cap K$, and suppose for $z \in D$,

$$
\|z-T(z)\| \leqslant\|x-T(x)\| \text { for all } x \in \partial_{K} B \text {. }
$$

Then the sequence $\left\{T^{n}(z)\right\}$ lies in $D$ and converges to a fixed point of $T$.

Proof. (3) Let $y$ be a point for which condition (3) is satisfied and, for convenience, assume $T(y)=0$. Then (3) implies

$$
\|x\|>\|y-x\| \geqslant\|T(x)\| \text { for all } x \in \Delta^{\prime} X,
$$


and this in turns implies $0 \notin \Delta^{\prime} X$ and it also implies

$$
T(x) \neq \lambda x \text { for all } x \in \Delta^{\prime} X \text { and } \lambda>1 \text {. }
$$

Since $0 \notin \Delta^{\prime} X$ there exists $\rho>0$ such that $B(0 ; \rho) \cap X$ is convex, and for $t>1$ sufficiently large, $t^{-1} T: B(0 ; \rho) \cap X \rightarrow B(0 ; \rho) \cap X$. Thus

$$
H=\left\{t \geqslant 1: T\left(x_{t}\right)=t x_{t} \text { for some } x_{t} \in X\right\} \neq \varnothing .
$$

Since $X$ is compact and $T$ continuous, $H$ is obviously closed in $[1, \infty)$. To see that $H$ is also open in $[1, \infty)$ suppose $r \in H$ with $x_{r} \in X$ satisfying $T\left(x_{r}\right)=r x_{r}$. Clearly we may suppose $r>1$. By $(*), x_{r} \notin \Delta^{\prime} X$, so for $\rho>0$ sufficiently small, $D=$ $B\left(x_{r} ; \rho\right) \cap X$ is convex. Moreover, since $r^{-1} T$ is a contraction mapping defined on $D$ having $x_{r}$ as its unique fixed point,

$$
\delta=\inf \left\{\left\|x-r^{-1} T(x)\right\|: x \in D,\left\|x-x_{r}\right\|=\rho\right\}>0 .
$$

Hence for $t>1$ sufficiently near $r$,

$$
\left\|x-t^{-1} T(x)\right\|>\delta / 2 \text { for } x \in D,\left\|x-x_{r}\right\|=\rho,
$$

and

$$
\left\|x_{r}-t^{-1} T\left(x_{r}\right)\right\| \leqslant \delta / 2 \text {. }
$$

By Proposition 1 there exist, for such $t$, points $x_{t} \in D$ such that $x_{t}=t^{-1} T\left(x_{t}\right)$. Thus $H$ is open in $[1, \infty)$ and the proof is complete.

\section{REFERENCES}

1. N. A. Assad and W. A. Kirk, Fixed point theorems for set-valued mappings of contractive type, Pacific J. Math. 43 (1972), 553-562.

2. E. Chandler. Fixed points and boundaries, Proc. Amer. Math. Soc. 82 (1981), 398-400.

3. C. M. Dafermos and M. Slemrod, Asymptotic behavior of nonlinear contraction semigroups, J. Funct. Anal. 13 (1973), 97-106.

4. R. De Marr, Common fixed-points for commuting contraction mappings, Pacific J. Math. 13 (1963), 1139-1141.

5. H. Freudenthal and W. Hurewicz, Dehnungen, Verkurzungen, Isometrien, Fund. Math. 26 (1936). 120-122.

6. W. A. Kirk, Locally nonexpansive mappings in Banach spaces (Proc. Workshop Fixed Point Theory. Univ. de Sherbrooke. June 1980), Lecture Notes in Math., vol. 886, Springer-Verlag. Berlin and New York, 1981.

Department of Mathematics, University of Iowa, Iowa City, Iowa 52242 